\title{
Public Information System Interface Design Research
}

\author{
Ning Zhang, Junliang Chen, Zhengjie Liu, and Jun Zhang \\ Computer Science and Technology College, Dalian Maritime University, \\ 116026 Dalian, China \\ \{jenny, liuzhj, chenjl, william\}@dlmu.edu.cn
}

\begin{abstract}
The diversity of users' cognitive skills remains the challenge of public information system interface design. In this paper, we focus on the universal interaction design method for public information systems like kiosks. We have developed a method with six steps based on the resources model. The method we proposed aims at reducing users' cognitive load and enabling designers to optimize interface information. To validate this method, two prototypes were designed based on the method and a usability test was conducted to compare users' cognitive load, performance and satisfaction between the designed prototypes and the current referencing system. Results show that, in contrast with the current reference system, prototypes we designed based on the proposed method can reduce user's cognitive load, and enhance user's performance and satisfaction.
\end{abstract}

Keywords: Universal usability, Cognitive load, Public information system.

\section{Introduction}

With the widespread of public information systems (PIS) in various service industries in China, users with various backgrounds become a challenge for PIS's interface design [1]. PIS designers and interface researchers have been exploring different ways to provide PIS users with more user-friendly and understandable interaction platform, including developing multi-model interface [2], setting up accessibility guidelines [3], and providing interface solutions based on usability test [4]. However, the lack of interaction design theory and design methods concerning PIS users 'cognitive levels remains the bottleneck for PIS interaction design, resulting in the loss of users and the low use rate of current PIS in China.

In this paper, a PIS interface design model based on distributed cognition theory was built to provide foundations and guide for the appropriate PIS interface design method, so that users' cognitive load can be minimized during the PIS interface design process. With hotel self-service kiosk interface design as an example, two prototypes were designed based on the proposed PIS interface design method. To validate whether PIS interface design method can reduce users' cognitive load, a comparative usability test was carried out to evaluate users' performance, satisfaction and cognitive load. Results confirm the validity and effectiveness of the proposed PIS interface design method. 


\section{Relevant Research}

Resources model of distributed cognition theory and universal design method are main supportive research area for this study.

\subsection{Resources Model}

Resources model of distributed cognition theory was first introduced into humancomputer interaction (HCI) design by Wright [5], to describe how information is distributed between users and computer systems, how to design appropriate and reasonable outer representations to minimize users' cognitive load. Resources model is mainly applied to describe human computer interaction activities and evaluate interfaces, and remains one of the most influential models in HCI field.

Resources refer to a collection of information structures that can be defined for each step in an interaction and which can be used to inform action. Information structure and interaction strategies are two components of resources model. Plan following, plan construction, goal matching and history-based selection are four strategies defined by Wright to analyze, design and evaluate interfaces. The basic information structure of resources model consists of six elements, including plan, goal, affordance, history, state and action-effect relations. Detailed definitions of the six elements are listed in figure 1. As an emerging theory in HCI, resources model is mainly applied in HCI research by Smith [6], Cheng [7] and Wang [8].

Table 1. Elements definitions of information struction of resources model

\begin{tabular}{|l|l|}
\hline \multicolumn{1}{|c|}{ Element } & \multicolumn{1}{c|}{ Definition } \\
\hline Plan & A sequence of actions, events and states that could be carried out. \\
\hline Goal & A required state of the system \\
\hline Affordance & $\begin{array}{l}\text { A set of possible next actions can be taken by the user for a give } \\
\text { state of the system }\end{array}$ \\
\hline History & Actions, events or states already achieved in the interaction \\
\hline Sate & $\begin{array}{l}\text { The collection of relevant values of the objects that feature in the } \\
\text { interaction at a given point in the interaction }\end{array}$ \\
\hline Action-effect relation & $\begin{array}{l}\text { A causal relation between an action or event and state which } \\
\text { represents the effect that executing the action or event will have on } \\
\text { the interaction }\end{array}$ \\
\hline
\end{tabular}

\subsection{Universal Usability}

Universal usability was first put forward in interface interaction design area by Ben Schneiderman [9], emphasizing the design of information products and services should be usable for every citizen.

Universal usability design (UUD) method includes three steps. First, it requires designers to classify different user groups that need special considerations during 
design process; second, build universal usability design matrix for the classified user groups, listing the specific requirements of different user groups and relevant design items; third, propose design solutions according to design matrix and evaluate the design solutions.

Since PIS users significantly differ in terms of age, education background, and other variables impacting cognitive load, it is feasible to adopt universal usability design method to guide PIS design process. As a newly introduced design method in HCI study, the application of this method in PIS interaction design is still limited, some representative exploring studies include Kouroupetroglou [10], Carbonell [11], and Takeo [12].

\section{Interaction Model for PIS}

There are two common ways to reduce users' cognitive load in interaction process. One is to simplify interaction flow; the other is to provide information easily understood for majority users. Resources model and UUD method can provide necessary support for the realization of the above mentioned ways. By applying resources model into the development of PIS interface, interaction flow can be simplified during design process, and cognitive resources can be allocated to systems as much as possible. Combing with UUD method, designers can provide appropriate information easily understood for majority users. Based on resources model and UUD method, PIS interface interaction model and design method can be built to help designers make reasonable interface information allocation decisions.

\subsection{PIS Interface Information Structure}

PIS interface differs from traditional desk interface in its full screen display, and users can only complete tasks in a certain fixed interaction flow. Besides that, public context has impact on users' interactions with PIS. Therefore, we adopt flow and context as two key elements into PIS interface information structure. Flow is defined as the set of actions during interaction process. Describing flow can help interface

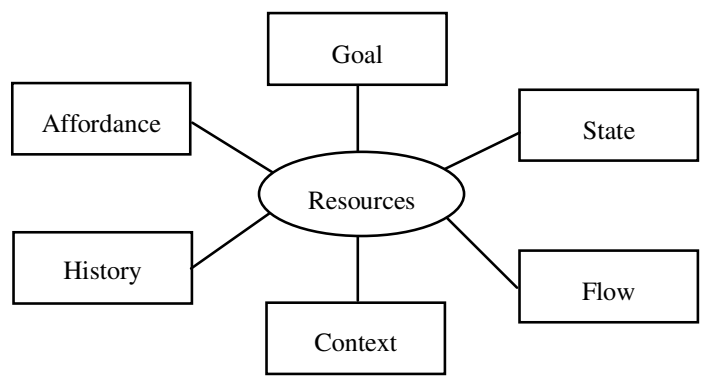

Fig. 1. PIS interface information structure 
designers and researchers follow users' real time goals. Context is defined as users' action-related conditions and variables in human computer interaction. The illustrations of PIS interface information structure is showed in figure 1.

The PIS interface information structure in fig 1 is aimed at describing general information a user need to complete a certain task through PIS. Based on this information structure, a set of interaction strategies can be built to guide the design and evaluations of PIS interaction process.

\subsection{PIS Interface Interaction Strategy}

We propose three basic interaction strategies for PIS interface, including goal matching and optimization, flow construction and evaluations, and affordance identification and obtaining. These interaction strategies can describe users' possible actions and correspondent operations a system provides in interaction process.

(1) Goal matching and optimization

According to goal matching and optimization strategy, users need goal, context, flow, state, and affordance to complete a task. This is a dynamic process. The state of resources changes with users' operations. For users with different cognitive skills, the matching extent of goals and resources differs. Designers have to analyze users' cognitive skills and evaluate the resources a system can provide to improve design and realize the optimized matching of users' goals and resources a system can provide. Detailed goal matching and optimization strategy is showed in figure 2.

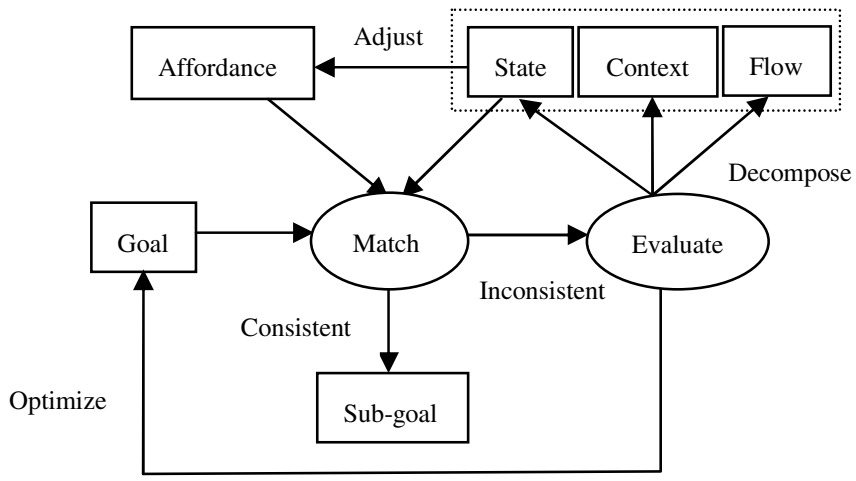

Fig. 2. Goal matching and optimization strategy

This strategy requires designers investigating users' cognitive skills before allo cating resources during interface design process. Methods like card sorting and participatory design can help designers bridge the gap between users' mental model and designers' mental model, and realize goal matching in an optimum condition.

(2) Flow construction and implementation

Users need information like affordance, current system state to decide next possible actions when interacting with computers. Basic operation flow of a task is 
composed of necessary interaction steps. Efficient and concise operation flow will facilitate the realization of goals. Detailed flow construction and implementation strategy is showed in figure 3 . In figure 3 , internal flow means the task completion order users perceive according to knowledge and system current state, external flow is the real task completion order of the designed system. Designers need analyze users' understanding about the system's working flow and decide the optimum flow for system development.

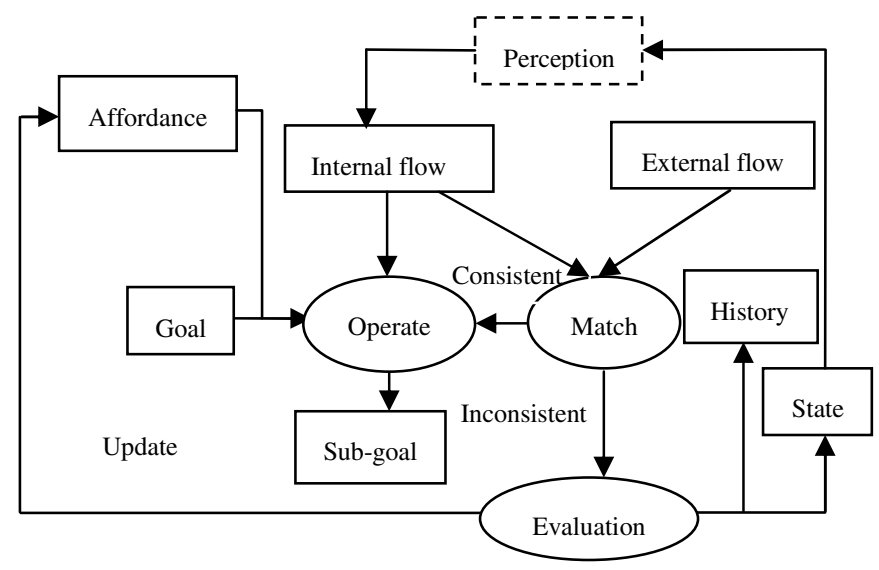

Fig. 3. Flow construction and implementation

This strategy requires designers building the appropriate task completion order and interface layout based on interaction task analysis. Universal usability design method can be taken into account to design interface layout. The design matrix from UUD method includes all the affordance users of different groups need to take actions according to task completion order. The set of affordance in the design matrix lists necessary points designers need consider in interface design process. PIS interface designers can make use of UUD method to propose specific design proposals for users with different background.

(3) Affordance identification and obtaining

Whether or not obtaining the right affordance is crucial for users to complete an interaction task. Too much affordance will increase users' cognitive load, while the lack of affordance prevents users completing tasks. Due to the diversity of PIS users' background, different users need different affordance. Therefore, affordance identification and obtaining strategy requires designers evaluating users' cognitive load and adjusting affordance based on evaluation results. Besides interface layout, context is also an important factor influence users' affordance identification and obtaining. If users' cognitive load is tested in usability lab, designers can use tapping test to simulate real operation situations, and to enhance the reliability of evaluation results. 


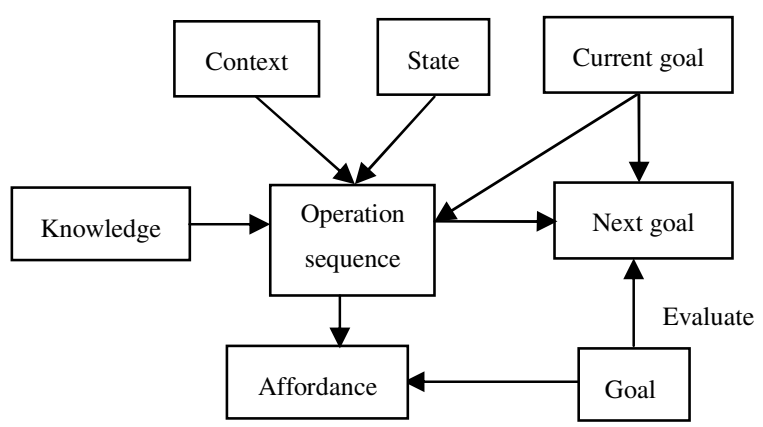

Fig. 4. Affordance identification and obtaining

The above mentioned PIS interaction model reflects the relationship between actions and information presentations. However, only models are not enough for interface designers to carry out specific design. PIS interaction methods based on PIS interaction model are necessary tools for designers to operate during design process.

\section{PIS Interaction Design Method}

Goal matching and optimization strategy requires designers focusing on different user groups' cognition analysis; flow construction and implementation strategy requires designers implementing information architecture under the help of UUD method; affordance identification and obtaining strategy requires designers evaluating users' cognitive load to make sure if the design proposal is reasonable enough for users with diversified background. These strategies need to be implemented throughout the whole design process. The common user-centered design methods include user needs analysis, functional analysis, information architecture, prototype design and usability evaluation. Correspondently, the PIS interaction design method we proposed is divided into six stages, that is, users' cognitive analysis, interaction task analysis, interface information layout, design project framing, prototype design, and cognitive load evaluation. Figure 5 shows the outline of the PIS interaction design method.

\subsection{Users' Cognitive Analysis}

Most of PIS users are either beginners or occasionally users, named "forever middle users" by Alan Cooper [13], not to mention those without computer skills. Relevant research [1] shows that age, education and experience are main factors influencing users' attitudes towards PIS products. Therefore, PIS users can be grouped according to their age, education and PIS product experience.

After dividing users into different groups, designers need contextual inquiry and interview to analyze different users' cognition towards PIS products, watching or interviewing users about their interaction experience with PIS products, recording the problems they meet, and the reasons users' goals are not matched, including problems from contextual influence, information overload, information misleading, 
and information deficiency. According to ISO25062 [14], each user group need at least 8 participants. Findings from users' cognition analysis should be edited into table form, as checklists for further evaluation reference.

\subsection{Interaction Task Analysis}

In interaction task analysis stage, designers need solve two problems. One is task categorization; another is to ensure task completion orders. The aim of this stage is to settle the general structure of the system being designed, and to describe the relationship between users' input and system's output.

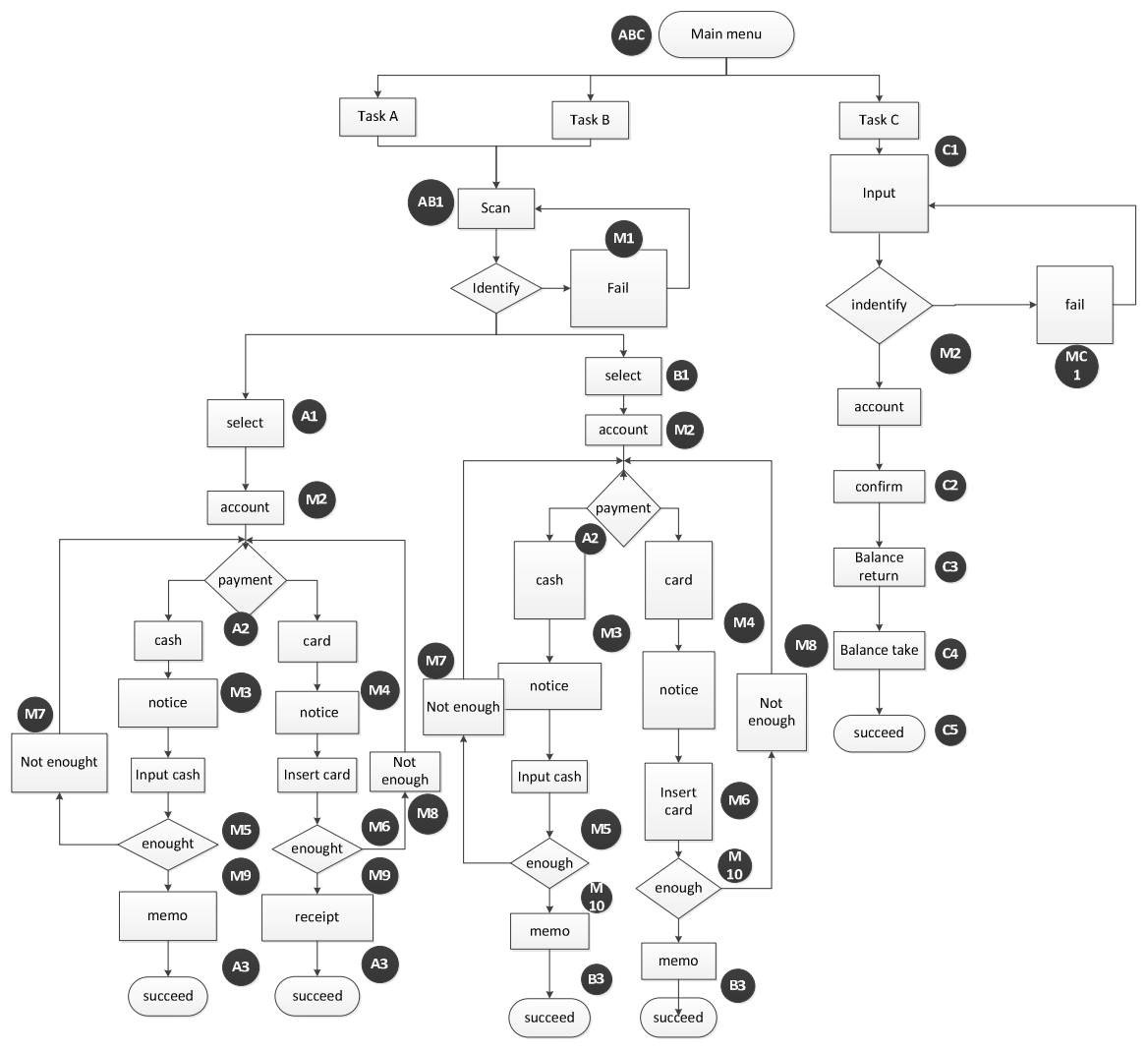

Fig. 5. Illustrations of task completion order

Designers can categorize different tasks need to be realized in the target system by card sorting, and then build completion order for each specific task through participatory design method, each step of the task flow has to be numbered according to the fixed order, as it is showed in figure 5. In this way, the basic task flow of the system can be drawn. In figure 5, the work flow of the three tasks of the main menu is listed. 
For example, $\mathrm{C} 1$ in figure 5 represents the first subtask of task $\mathrm{C}$, and M2 represents the second system information provided for users to complete certain task.

\subsection{Interface Information Layout}

Based on the results of users' cognition analysis and interaction task analysis, PIS interface design matrix can be built through UUD method. All the points listed in the matrix can help designers do specific design to reduce users' cognitive load, as it is showed in table 2 .

Table 2. Examples of design matrix

\begin{tabular}{|c|c|c|c|}
\hline sk & Senior users & Fresh users & Low educated users \\
\hline Step 1 & $x x$ & $x x$ & $x x$ \\
\hline Step 2 & $x x$ & $x x$ & $x x$ \\
\hline Step 3 & $x x$ & $x x$ & $x x$ \\
\hline$:$ & $x x$ & $x x$ & $x x$ \\
\hline : & $x x$ & $x x$ & $x x$ \\
\hline Step n & $x x$ & $x x$ & $x x$ \\
\hline
\end{tabular}

With user groups as the horizontal axis of the matrix, and task completion order as the vertical axis, designers can input the rest intersectional content of the matrix according to users' cognition analysis results, and current PIS interface design standards [3], including various specific requirements of different user groups, the solutions of their problems need to be considered. Each solution corresponds to the problem a group of user have in a certain stage of a task flow. Usually there is no appropriate data that can be used directly by designers in a specific design process, especially quantitative data. Users' self-reported data obtained by interview, observation and contextual inquiry is the main source for designing PIS interface for users with different cognitive levels.

\subsection{Design Project Framing}

The output of design project framing is design document. Interaction designers need to draw up specific design documents based on the design matrix, so that system developers can read in details. A basic design document is consisted of information architecture, interface layout, interface elements, task completion flow and interface order.

\subsection{Prototype Design}

In prototype design stage, designers can use Flash, Axure RP and other tools to realize the information visualization based on the design documents. The designed 
prototypes should be revised according to evaluation results. Formative evaluation methods like cognitive walkthrough and heuristic evaluation can be applied in this stage to save design and development cost.

\subsection{Cognitive Load Evaluation}

User's cognitive load level correlates the extent of system affordance identification and obtaining. Research has found that users' performance is not directly correlated with cognitive load level, some system are tested with high performance but low users' satisfaction [15]. The main reason is the existence of cognitive load during human computer interaction process. Therefore, it is necessary to evaluate the cognitive load level of PIS users.

Tapping test proves an effective and efficient method to test users' cognitive load and system's usability [16]. Comparison test is also necessary for designers to compare users cognitive load levels when interacting with different prototypes. Cognitive load can be evaluated by NASA-TLX [17], Paas Scale [18], and user performance.

\section{Case Study}

In order to test the validity and feasibility of the proposed PIS interaction method, we recruited two interaction designers to design hotel self-service system interface according to the requirements of PIS interaction design method. We chose PIS in hotel industry because of the increasing tendency of PIS applications in hotels, and the diversity of hotel clients.

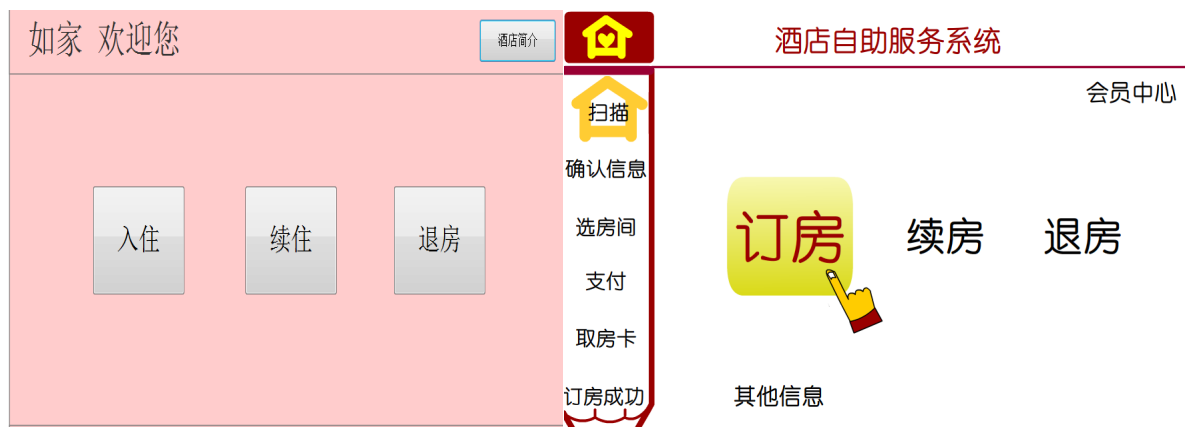

Fig. 6. Prototype A

Fig. 7. Prototype B

Prototype system A and B designed by PIS interaction design method are showed in figure 6 and figure 7 . We compare the prototypes with a current running hotel self-service system in Shandong, China, as the reference system showed in figure 8. Prototype systems and the reference system will be compared in terms of users' cognitive load, performance and satisfaction. 
The comparison test is conducted in the usability lab of Dalian Maritime University, China. We recruited 32 users to participate in our test, and divide these users in two groups. One is mainstream user group, with 16 users using PIS at least once a month, aged from 19 to 28 , all the users in mainstream user group are university students; the other is non-mainstream user group, with 16 users no PIS product experience, aged from 45 to 78 , all the users in testing group have no bachelor degree.

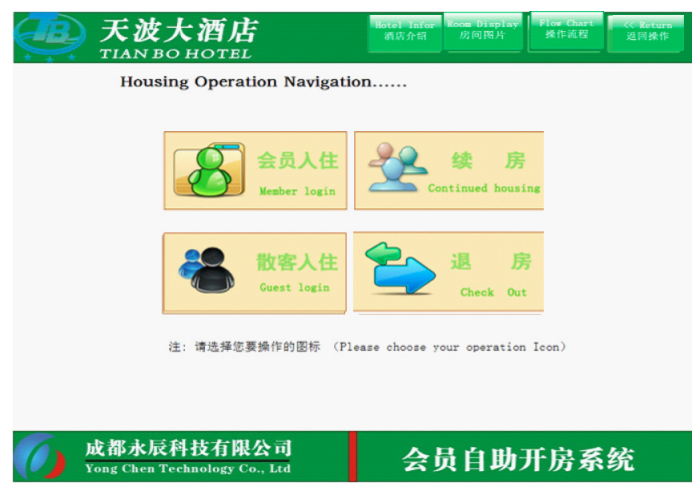

Fig. 8. Reference System

All users are required to complete three tasks on touchscreens, including self-service check-in, self-service check-out, and room renewal, using prototype system A and B, as well as reference system, When interacting with systems, users are asked to tap with the rhythm of the music playing in the usability lab. Users' interaction process is recorded to calculate task completion time and error rate. Users are also asked to complete NASA-TLX scale and SUS (system usability scale) to calculate their cognitive load and satisfactions towards different systems. Test results are listed below.

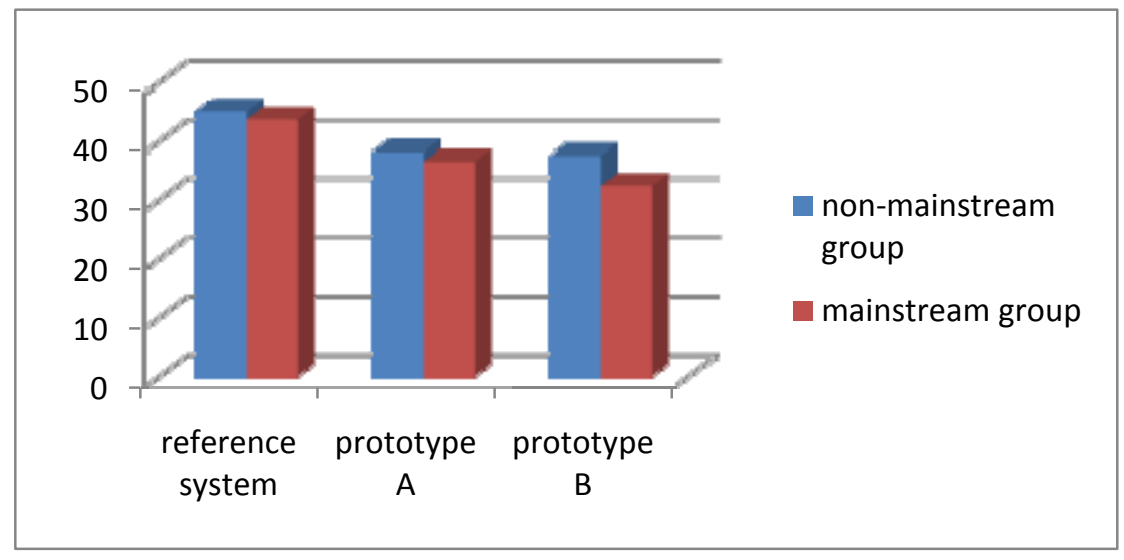

Fig. 9. Comparison of cognitive load means 
Figure 9 shows that the cognitive load of the mainstream user group is lower than the cognitive load of the testing group when they complete the designed tasks through the three systems. Both of the groups' cognitive load is lower when using prototype systems than using the reference system.

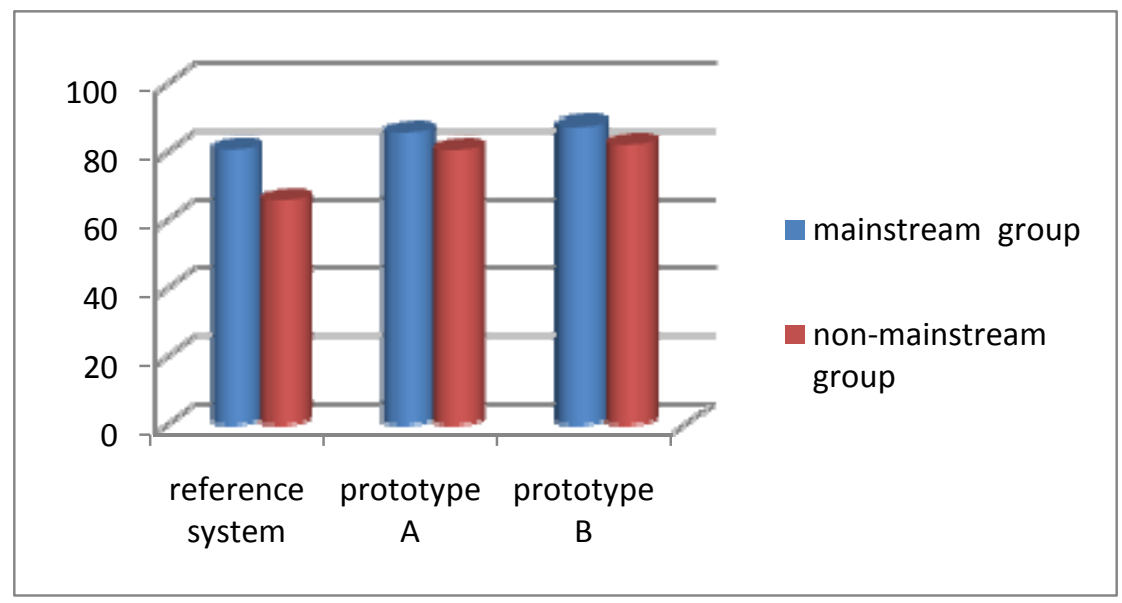

Fig. 10. Comparison of satisfaction means

Figure 10 shows that the mainstream users' satisfaction towards the three systems is higher than non-mainstream group's satisfaction. Both of the groups' satisfaction towards prototype systems is higher than towards reference system.

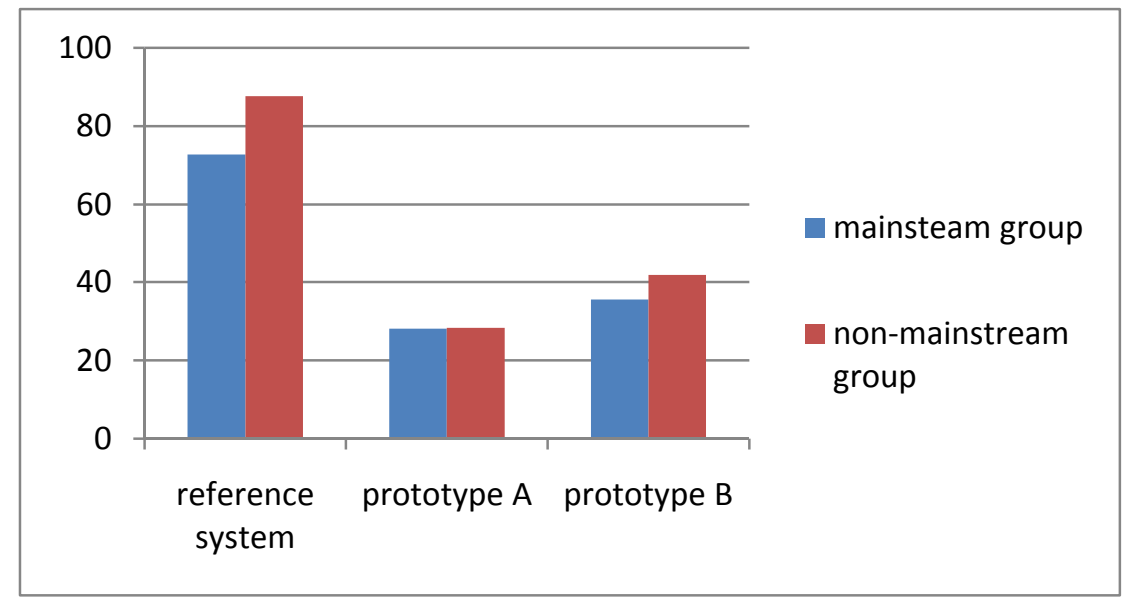

Fig. 11. Comparison of task completion time means 
Figure 11 shows that main stream users spend less time completing the designed tasks through three systems than non-mainstream users. Both of the groups spend less time completing the designed tasks through prototype systems than the reference system.

Tapping test results show that the ratios of mainstream users and non-mainstream users' missing the rhythm of the music when completing the designed task through the three systems are8:6, 4:3, and 1:1 respectively, as it is showed in figure 12.

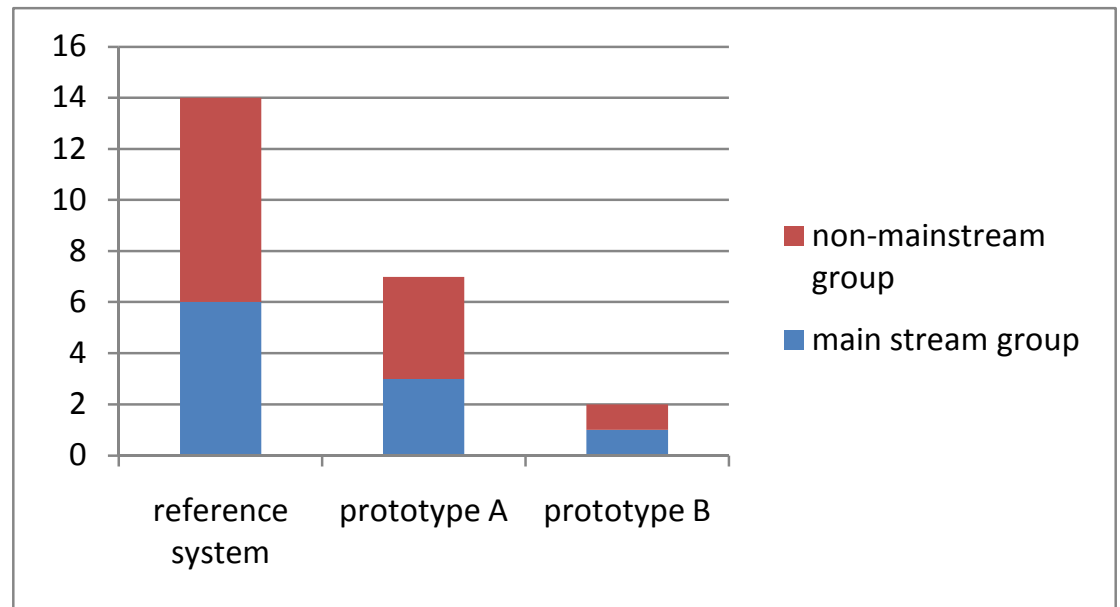

Fig. 12. Tapping missing times

Independent T test results show that there is significant difference between users' satisfaction towards reference system and towards prototype systems. There is also significant difference between users' performance on the reference system and on the prototype systems. However, there is no significant difference between users' cognitive load on the reference system and on prototype systems, and this result needs further validation among broader and more representative sample.

Data analysis results mentioned above, to some extent, support the validity and feasibility of the PIS interaction method.

\section{$6 \quad$ Limitations and Recommendations}

Due to the limitations of the research sample, the results of the study still need further validation. The comparison test was conducted in usability lab, although tapping test was applied to simulate the real interaction environment, there is still difference between lab environment and the real world situation. 


\section{References}

1. Cremers, A.H.M., de Jong, J.G.M., van Balken, J.S.: User-Centered Design with Illiterate Persons: The Case of the ATM User Interface. In: Miesenberger, K., Klaus, J., Zagler, W.L., Karshmer, A.I. (eds.) ICCHP 2008. LNCS, vol. 5105, pp. 713-720. Springer, Heidelberg (2008)

2. Mark, T., Fredrik, E., Annika, W.: Adaptive User Interfaces for Public Kiosks. Journal of User Modeling and User-Adapted Interaction 14(2) (2004)

3. Maguire, M.C.: A Review of User-Interface Design Guidelines for Public Information Kiosk Systems. International Journal of Human-Computer Studies 50, 263-286 (1999)

4. Farooq, A.: Haptics in Kiosks and ATMs for the Disabled. University of Tampere, Tampere (2009)

5. Wright, P.C., Fields, R.E., Harrison, M.D.: Analyzing Human-Computer Interaction as Distributed Cognition: the Resources Model. Journal of Human Computer Interaction 51, 1-41 (2001)

6. Smith, S., Duke, D., Wright, P.C.: Using the Resources Model in Virtual Environment Design. In: King's Manor Workshop on user-Centered Design and Implementation of Virtual Environments. University of York (1996)

7. Cheng, S.W., Sun, S.Q.: Human-Computer Interaction Resource Model based on Distributed Cognition. Journal of Computer Integrated Manufacturing System 14, 1683-1703 (2008)

8. Wang, C.Q., Deng, C.Z., Ma, C.X., et al.: an Extended Resources Model based on Distributed Cognition. Journal of Software 16, 1717-1725 (2005)

9. Shneiderman, B., Hochheise, H.: Universal Usability as a Stimulus to Advanced Interaction Design. Behaviour \& Information Technology 20, 367-376 (2001)

10. Kouroupetroglou, G.: Universal Access in Pubic Terminals: Information Kiosks and Automated Teller Machines. In: The Universal Access Handbook. CRC Press (2008)

11. Carbonell, N., Stephanidis, C.: Universal Access: Theoretical Perspectives, Practice and Experience. In: 7th ERCIM International Workshop on User Interfaces for All, pp. 63-67 (2002)

12. Takeo, A., Akinori, O., Taku, A.: Promoting Universal Design of Automated Teller Machines. FUJITSU Scientific and Technical Journal 41, 86-96 (2005)

13. Cooper, A., Ding, C., Dream, D.: The Inmates are Running the Asylum: Why High-Tech Products Drive Us Crazy and How to Restore the Sanity. Publishing House of Electronics Industry, Beijing (2006)

14. ISO25062.Software Product Quality Requirements and Evaluation (SQuaRE)-Common Industry Format (CIF) for Usability Test Reports. International Standards Organization. Geneva, Switzerland (2006)

15. Fisher, C.D.: Why do Lay People Believe that Satisfaction and Performance are Correlated? Possible Sources of a Commonsense Theory. Journal of Organizational Behavior 24, 753-777 (2003)

16. Albers, M.: Tapping as a Measure of Cognitive Load and Website Usability. In: Proceedings of the 29th ACM International Conference on Design of Communication, pp. 25-32 (2011)

17. Hart, S.G.: NASA- Task Load Index (NASA-TLX):20 Years Later. In: Proceedings of the Human Factors and Ergonomics Society 50th Annual Meeting, pp. 904-908 (2006)

18. Paas, F., Tuovinen, J.E., Tabbers, H., et al.: Cognitive Load Measurements as Means to Advance Cognitive Load theory. Educational Psychologist 38, 63-71 (2003) 